\title{
The LCOGT NEO Follow-up Network
}

\author{
Tim A. Lister ${ }^{1}$, S. Greenstreet ${ }^{1}$, E. Gomez ${ }^{1}$, E. Christensen ${ }^{2}$ and \\ S. Larson ${ }^{2}$ \\ ${ }^{1}$ Las Cumbres Observatory Global Telescope (LCOGT), \\ 6740 Cortona Drive Suite 102, Goleta, CA 93117, USA \\ email: tlister@lcogt.net \\ ${ }^{2}$ Catalina Sky Survey, Lunar and Planetary Laboratory, University of Arizona, \\ 1629 E University Blvd Tucson, AZ 85721-0092, USA
}

\begin{abstract}
Las Cumbres Observatory Global Telescope Network (LCOGT) has deployed a homogeneous telescope network of nine 1-meter telescopes to four locations in the northern and southern hemispheres, with a planned network size of twelve 1-meter telescopes at 6 locations. This network is very versatile and is designed to respond rapidly to target of opportunity events and also to perform long term monitoring of slowly changing astronomical phenomena. The global coverage of the network and the apertures of telescope available make LCOGT ideal for follow-up and characterization of Solar System objects (e.g. asteroids, Kuiper Belt Objects, comets, Near-Earth Objects (NEOs)) and additionally for the discovery of new objects.

We are using the LCOGT network to confirm newly detected NEO candidates produced by the major sky surveys such as Catalina Sky Survey (CSS) and PanSTARRS (PS1\&2) and several hundred targets are now being followed per year. An increasing amount of time is being spent to obtain follow-up astrometry and photometry for radar-targeted objects and those on the NearEarth Object Human Space Flight Accessible Targets Study (NHATS) or Asteroid Retrieval Mission (ARM) lists in order to improve the orbits, determine the light curves and rotation periods and improve the characterization. This will be extended to obtain more light curves of other NEOs which could be targets. Recent results have included the first period determinations for several of the Goldstone-targeted NEOs. We are in the process of building a NEO followup portal which will allow professionals, amateurs and Citizen Scientists to plan, schedule and analyze NEO imaging and spectroscopy observations and data using the LCOGT Network and to act as a co-ordination hub for the NEO follow-up efforts.
\end{abstract}

Keywords. astrometry; minor planets, asteroids; telescopes; techniques: photometric

\section{Introduction}

Near Earth Objects (NEOs) are our closest neighbors and research into them is important not only for understanding the Solar System's origin and evolution, but also to understand and protect human society from potential impacts. NEOs originate in collisions between bodies in the main asteroid belt and have found their way into near-Earth space via complex dynamical interactions. Understanding these interactions, the populations and the orbital element distribution requires accurate orbits and complete samples for the NEO population, in order to properly debias the sample and correctly model the NEO population. The existing surveys such as the PanSTARRS1 (PS1) \& PanSTARRS2 (PS2) surveys (Wainscoat et al. 2014), NEOWISE (Mainzer et al. 2014), and to a lesser extent, the Catalina Sky Survey (CSS), are not normally capable of following-up their NEO candidate detections and require additional programs of NEO follow-up on other telescopes to confirm and characterize the new NEOs.

LCOGT has deployed a homogeneous telescope network of nine 1-meter telescopes to four locations in the northern and southern hemispheres. The global coverage of this 


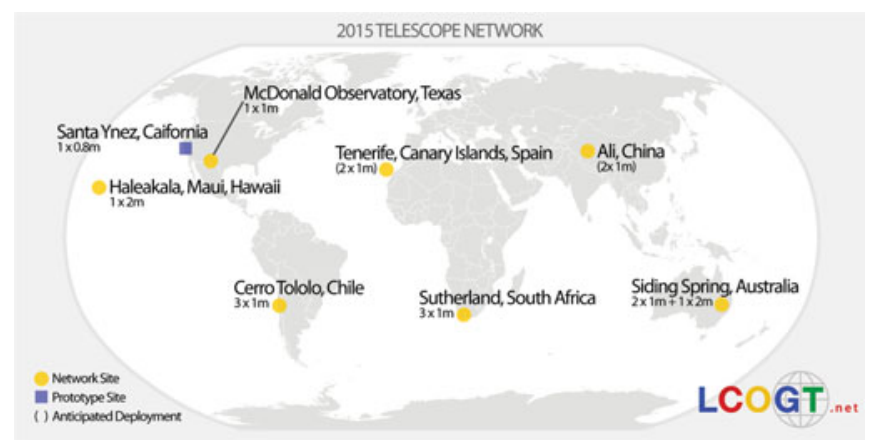

Figure 1. Network map of LCOGT facilities.

network and the apertures of telescope available make LCOGT ideal for follow-up and characterization of Solar System objects in general and for Near-Earth Objects (NEOs) in particular.

\section{Overview of the LCOGT Network}

LCOGT completed the first phase of the deployment (see Figure 1) with the installation and commissioning of the nine 1-meter telescopes at McDonald Observatory (Texas), Cerro Tololo (Chile), SAAO (South Africa) and Siding Spring Observatory (Australia). These 1-meter telescopes join the existing two $2 \mathrm{~m}$ Faulkes Telescopes which LCOGT has operated since 2005. The whole telescope network has been fully operational since 2014 May, and observations are being executed remotely and robotically. Future expansion to sites in the Canary Islands and Tibet are planned for 2016.

The $2 \mathrm{~m} \mathrm{FTN}$ and FTS telescopes have a $10^{\prime} \times 10^{\prime}$ FOV CCD imager with 18 filters, high-speed camera and a low-resolution $(R \sim 400,320-1000 \mathrm{~nm})$ FLOYDS spectrograph. The $1 \mathrm{~m}$ telescopes will have a $26^{\prime} \times 26^{\prime}$ FOV CCD with 21 filters and high-speed cameras and will also have fiber feeds to a high-resolution $(R \sim 53,000)$ NRES echelle spectrograph (Eastman et al. 2014) (one per site). More details are given in Brown et al. (2013).

\section{Use of the LCOGT Network for NEO follow-up}

\subsection{NEO Follow-up for PS1 \& 2, CSS, NEOWISE \& others}

To handle the large number of NEO candidates that the surveys produce (thousands per year) and ensure they get follow-up confirming astrometry and photometry, we have developed a new automated system called "NEOexchange" $\dagger$. This system can retrieve new NEOs (from the MPC or Goldstone), compute orbits, plan observations and automatically schedule them for follow-up on the robotic telescopes of the LCOGT Network.

This builds on our previous efforts (e.g. Lister 2014) but uses more modern web development and deployment frameworks such as Django and Docker to make a redeployable web service that can support many users and multiple projects that are using the LCOGT Network. This will include an extension to support citizen scientists in the future.

\subsection{Follow-up of radar-targeted NEOs and Potential Mission Destinations}

Radar observations are a very powerful tool that are needed to spatially resolve NEO targets, determine binary fraction and improve orbits, preventing loss and impact risk

$\dagger$ http://lcogt.net/neoexchange/ 

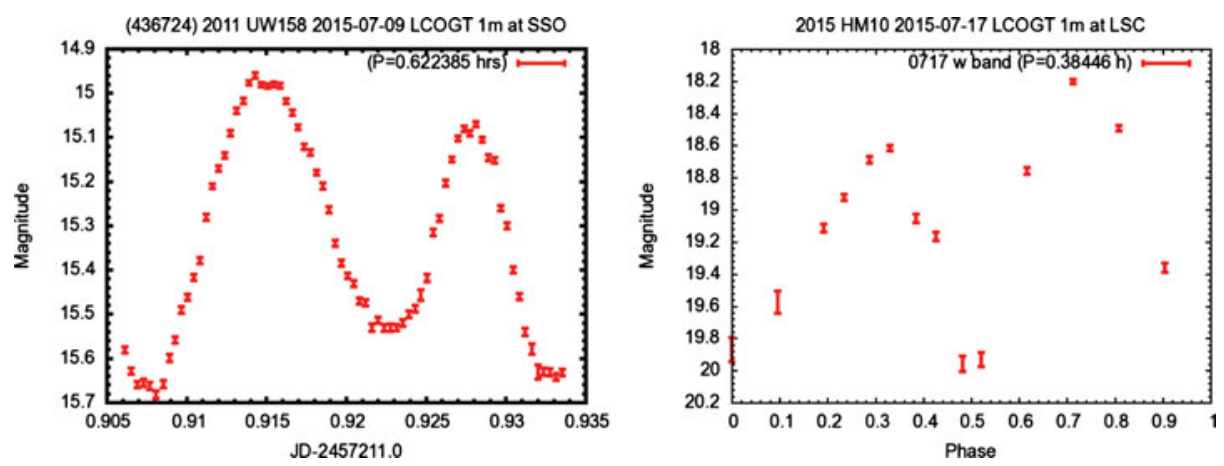

Figure 2. Light curve (left) from LCOGT of (436724) 2011 UW158. The $\sim 450 \mathrm{~m}$ NEO has an elongated shape, with a rotational variation of $\sim 0.7 \mathrm{mag}$. We determined a rotation period of $\sim 36$ minutes. (Right) Light curve from LCOGT of 2015 HM10. The $\sim 60 \mathrm{~m} \mathrm{NEO} \mathrm{has} \mathrm{a} \mathrm{very}$ elongated shape, as indicated by the $\sim 1.5 \mathrm{mag}$ amplitude. The light curve is phased on the rotation period of $\sim 23$ minutes.

assessment (e.g. Ostro et al. 2007). The LCOGT NEO Follow-up Network provides rapid response astrometry to allow pointing and imaging by radar assets such as Goldstone and Arecibo for targets that have only recently been discovered. The network can also obtain colors and photometric light curves which allow the determination of rotation rates, pole directions, spectral classes \& shapes. Examples of the follow-up photometry and period determinations obtained for two radar-targeted NEOs are shown in Figure 2.

Potential mission destinations such as NEO Human Space Flight Accessible Targets Study (NHATS) and Asteroid Retrieval Mission (ARM) targets are usually small in size with a limited visibility window of days to a few weeks. Rapid responses with the LCOGT network allows characterization with astrometry, photometry and eventually (robotic) spectroscopy.

\section{Results and Future Work}

Follow-up of NEO candidates with the LCOGT telescopes in the past 12 months has resulted in 11,900+ measurements on 2300+ objects and the confirmation of $250+$ new NEOs, 8 comets and 690+ other objects from Catalina, PS1, NEOWISE and other surveys. We are providing the bulk of the follow-up for PS1 and NEOWISE, particularly in the Southern Hemisphere. In the process of the follow-up, we have discovered over 55 new Main Belt objects and recovered objects as faint as $R \sim 23$ showing the potential of the $1 \mathrm{~m}$ network for NEO follow-up. We will be continuing to develop NEOexchange to allow use by external users (professional and Citizen Scientist), observation campaign planning and online data analysis and reporting.

\section{References}

Brown, T. M., Burleson, B., De Vera, J. et al. 2013, PASP, 125, 1031.

Eastman, J. D., Brown, T. M., Hygelund, J., et al. 2014, Proc. SPIE, 9147, 914716.

Lister, T. 2014, in: K. Muinonen et al. (eds), Proc. Asteroids, Comets, Meteors 2014, p. 320.

Mainzer, A., Bauer, J., Cutri, R. M., et al. 2014, ApJ, 792, 30.

Ostro, S. J., Giorgini, J. D. \& Benner, L. A. M. 2007, in: G.B. Valsecchi, D. Vokrouhlick, A. Milani (eds), Proc. IAU Symposium No. 236, p. 143.

Wainscoat, R., Veres, P., Bolin, B., et al. 2014, in: K. Muinonen et al. (eds), Proc. Asteroids, Comets, Meteors 2014, p. 574. 